\title{
EVALUACION DEL POTENCIAL DE APRENDIZAJE EN NIÑOS, Y ADOLESCENTES CON DISCAPACIDAD INTELECTUAL
}

\section{ASSESSMENT OF LEARNING POTENTIAL IN CHILDREN AND ADOLESCENTS WITH INTELLECTUAL DISABILITIES}

\author{
Ángela Magnolia Ríos Gallardo*, Lina Marcela Murcia Paredes**
}

\begin{abstract}
Resumen
El presente estudio tiene el objetivo de evaluar el potencial de aprendizaje de un grupo de 17 niños, niñas y adolescentes (NNA) de edades comprendidas entre los 8 y 18 años, diagnosticados con discapacidad cognitiva, pertenecientes a la fundación síndrome de Down y al colegio Utrahuilca de la ciudad de Neiva. El enfoque es cuantitativo con un diseño pre - experimental pretest y postest a grupo único. Inicialmente se procedió a la aplicación del Test de Inteligencia No Verbal TONI-2 para identificar dicho coeficiente de inteligencia. Este coeficiente se tiene en cuenta para tratar de ubicar a cada uno de los participantes en un nivel de inteligencia, debido a que todas las pruebas utilizadas en el estudio son no verbales. Posteriormente se administró el test de matrices progresivas Raven, series $\mathrm{A}, \mathrm{Ab}$ y $\mathrm{B}$, tanto en el pre-test como en el pos-test para evaluar el potencial de aprendizaje. En el intermedio entre pretest y postest se aplicó la Evaluación del Potencial de Aprendizaje EPA-2 con la metodología de la evaluación dinámica, tal como lo propone dicha prueba. Este test sirvió como entrenamiento para conocer el grado de modificabilidad cognitiva de los participantes y las estrategias utilizadas en la ejecución de la tarea propuesta.
\end{abstract}

Los resultados evidenciaron que para algunos participantes hubo una ganancia significativa, observado en los resultados favorables en el aprendizaje de una serie de procesos y operaciones ejecutivas requeridos en la resolución de este tipo de problemas. Mientras que otros sujetos, los cuales por su compromiso cognitivo moderado que involucra dificultad en la flexibilidad mental y capacidad de inhibición, además de dificultades atencionales y factores motivaciones, no se vieron beneficiados de este entrenamiento, sin embargo, esto no descarta que la incorporación de estrategias especificas y con un entrenamiento más amplio se logre estimular otras áreas y habilidades intelectuales.

Palabras clave: Discapacidad cognitiva, potencial de aprendizaje, modificabilidad cognitiva.

\section{Abstract}

The present study aims to assess the learning potential of a group of 17 children and adolescents (NNA) aged between 8 and 18 years, diagnosed with cognitive disabilities, members of the Down Syndrome Foundation and the school Utrahuilca in the city of Neiva. A quantitative approach was used, with a pre -

Artículo recibido: 04/04/2013 Aprobado: 30/06/2013

* Catedrática de la Facultad de Ciencias Sociales y Humanas. Email: neuro_angelamagnolia@hotmail.com

** Psicóloga. Joven investigadora Colciencias, 2011-2012. Email: limarc2701@hotmail.com 
experimental, pre-post test design study without a control group. Initially the Nonverbal Intelligence Test TONI-2 was applied to identify such IQ. This coefficient is taken into account to try to locate each of the participants in a level of intelligence, since all the tests used in the study are nonverbal. Subsequently, the Raven's Progressive Matrices test, series A, Ab and B, both in the pre-test and post-test was administered to evaluate the learning potential. In the time between pretest and posttest, the Learning Potential Assessment EPA-2 with the methodology of dynamic assessment, as proposed in the test, was applied. This test served as training to identify the degree of cognitive modifiability of the participants and the strategies used in the implementation of the proposed task.

The results showed that for some participants there was a significant gain, which is perceived in the favorable results in the learning of a series of executive operations and processes required in the resolution of problems of this type. While other individuals, who by their moderate cognitive impairment which involves difficulty in mental flexibility and ability of inhibition, in addition to difficulties with concentration and motivation factors, have not benefited from this training, however, this does not rule out that the incorporation of specific strategies and broader training will stimulate other areas and other intellectual skills.

Key words: cognitive disabilities, learning potential, cognitive modifiability.

\section{Introducción}

Para identificar la discapacidad cognitiva, anteriormente denominado retraso mental, históricamente los expertos se han centrado en la conceptualización y caracterización de la limitación significativa del funcionamiento intelectual, del comportamiento adaptativo y su comienzo antes de la edad adulta, (Vásquez, 2009), pero en la actualidad la Asociación Americana sobre Retraso Mental (AAMR) en 2002, la define como una discapacidad caracterizada por limitaciones significativas en el funcionamiento intelectual y la conducta adaptativa tal como se ha manifestado en habilidades prácticas, sociales y conceptuales de inicio previo a los 18 años de edad (Luckasson y cols., 2002, p. 8). Luego para atender a esta población, se tenía en cuenta únicamente el coeficiente de inteligencia para emitir un diagnostico, en la actualidad se identifican las habilidades adaptativas y se evalúan dichas habilidades en un contexto próximo y amplio.

Por lo tanto, este modelo de atención no reconocía en los niños, niñas y adolescentes (NNA) con discapacidad cognitiva las potencialidades para integrarlo a un contexto escolar y/o social, por el contrario sostenía argumentos para aislarlos del sistema educativo y de la sociedad, causando un efecto nocivo para el desarrollo de los procesos psicológicos superiores de los mismos, toda vez que estos están mediados por los signos y símbolos que son incorporados individualmente solo en las relaciones sociales, (Trilla, 2001).
Actualmente, se tiene una visión más multidimensional, donde se incluyen aspectos sociales y educativos como el aprendizaje, la participación, la interacción, los roles y las redes sociales de los NNA con esta discapacidad dentro de un marco de igualdad de oportunidades. Este modelo de atención plantea que la discapacidad cognitiva no es una condición sustantiva del individuo, ni un trastorno médico o mental, sino más bien un estado particular del funcionamiento que comienza en la infancia y su evolución está directamente relacionado con la disponibilidad de apoyos personalizados y efectivos para la integración sociocomunitaria, educativa y laboral, (Vásquez, 2009). La discapacidad cognitiva comenzó a concebirse hoy desde un enfoque que identifica al sujeto como a cualquier otro individuo de la sociedad sin ninguna otra distinción que la manera como se adapta y hace uso del contexto en el que se desenvuelve.

El surgimiento de esta nueva visión empieza a finales de los años ochenta, cuando la evaluación y el entrenamiento de la inteligencia se convierten en una de las áreas más importantes de estudio de la psicología cognitiva aplicada. No obstante, las aplicaciones prácticas de la investigación actual aún son escasas.

Hacia 1974, se desarrolló una teoría base de la modificabilidad cognitiva, junto con la idea de la experiencia de aprendizaje mediado con la que se proporciona al organismo instrumentos de adaptación y aprendizaje, a fin de hacer a la persona capaz de utilizar eficientemente 
la exposición directa al aprendizaje. La falta de experiencia de aprendizaje hace que el organismo continúe siendo un receptor pasivo de información, sin la necesidad de establecer relaciones entre acontecimientos y con muchas deficiencias en las fases de entrada, salida y elaboración del acto mental. (Feuerstein y col., 1974).

En los siguientes años, se han publicado artículos sobre nuevas alternativas a la evaluación tradicional de las habilidades cognitivas a partir de la implementación de las técnicas del potencial de aprendizaje (Budoff, 1975; Haywood, 1977; Fernández-Ballesteros, 1979; Sternberg, 1981). La evaluación del potencial de aprendizaje 0 evaluación dinámica, según las dos denominaciones más importantes que este tópico recibe actualmente, constituye una metodología de evaluación que surgió hace unos 30 años orientada inicialmente a la evaluación de la capacidad de aprender en niños que presentaban déficits intelectuales 0 de aprendizaje en los tests tradicionales de inteligencia, (Fernández y Fernández, 1990).

«Teóricamente, el concepto Potencial de Aprendizaje procede del formulado por Vygotsky como Zona de Desarrollo Próximo, definido como la distancia entre el nivel real de desarrollo, determinado por la capacidad de resolver independientemente un problema, y el nivel de desarrollo potencial, determinado a través de la resolución del problema bajo la guía de un adulto o en colaboración de otro compañero más eficaz», (FernándezBallesteros y col. (1987/1997).

Para Vigotsky (1978), la experiencia ha demostrado que el niño con una zona más amplia en su desarrollo próximo tendrá un mejor rendimiento escolar y, por tanto, la determinación de esta zona nos proporciona una clave más útil que la edad mental para descubrir la dinámica del progreso intelectual.

Por otra parte, para Calero y Márquez (1998), la evaluación del potencial de aprendizaje se refiere a «la evaluación de la capacidad general para aprender en respuesta a la instrucción de una tarea dada; 0, dicho de otro modo, la capacidad de un sujeto para sacar provecho de un entrenamiento dirigido a la enseñanza de resolución de problemas más o menos complejos en los que en un principio fracasa».

Según, Fernández-Ballesteros y Calero (1992), han considerado que son dos los objetivos claves de la Evaluación del Potencial de Aprendizaje: a. «El conocimiento de las estrategias, habilidades $u$ operaciones de control que requiere la resolución de un determinado problema y que subyace al proceso de aprendizaje».

b. «Profundizar en aspectos diferenciales (fundamentalmente deficitarios) del funcionamiento cognitivo con el fin de proceder y programar una intervención en ese ámbito».

El modelo de evaluación dinámica y el potencial del aprendizaje no utiliza contenidos académicos, en lugar de ello se dirige a entrenar e intentar modificar las funciones cognitivas considerando los estados emocionales de Ios NNA, a la hora de dar respuesta a una serie de tareas de características no verbales.

Dentro de este enfoque se propone (Feuerstein, Rand y Hoffman): 1) pasar de una orientación de producto a una de proceso, cambios que afectan a los métodos y técnicas, así como el tipo de preguntas e interpretación de las respuestas que se realizan, es decir <pedirle a Juan que se pare de la silla, vaya hacia el escritorio café y traiga el lapicero azul, de media vuelta, vaya nuevamente hacia la silla donde estaba sentado y me entregue el lapicero $>$ en vez de decirle < Juan tráigame el lapicero que está en el escritorio>; 2) cambiar de roles en la relación examinador examinado: el evaluador apoya y estimula al evaluado, de manera que le comunica como superar sus déficits en la solución de los problemas que se plantea; 3) modificar la estructura de la evaluación a un esquema test-entrenamiento-retest, lo que permite averiguar en qué medida se beneficia el examinado del aprendizaje, determinando así su potencial; y 4) modificar la interpretación de los resultados, resaltando los aspectos del proceso y no los aspectos cuantitativos.

De otro lado se debe tener presente que el lenguaje verbal es una limitación que está presente en esta población, por lo que se considera relevante revisar cómo el lenguaje no verbal, facilitaría las tareas para el aprendizaje de niños y niñas con necesidades educativas especiales, principalmente, en personas con discapacidad cognitiva. Se han realizado una serie de estudios que comprueban cómo el aprendizaje de niños con estas características se facilita con el uso de técnicas y estrategias que apuntan hacia la comunicación no verbal, o analógica, partiendo desde lo más procedimental (habilidades perceptivas, motoras y cognitivas adquiridas, no conscientes, relacionadas con la acción) hacia lo declarativo (más explícito, 
referido a las habilidades y hechos que se pueden expresar de manera consciente), y combinando el uso de lenguaje verbal con lenguaje no verbal (Seys, Kersten \& Duker, 1990; Romski \& Sevcik, 1997; Buffington, Krantz, McClannahan \& Poulson, 1998; Smith \& Bryson, 1998; Wetherby, Prizant \& Hutchinson, 1998; Jones \& Carr, 2004; Stephenson \& Dowrick, 2005). Por ejemplo, algunas investigaciones han permitido demostrar que los procesos de atención y memoria influyen en la interpretación y comprensión de la información en personas con discapacidad intelectual, observándose que el uso de la comunicación no verbal facilita dichos procesos (Drews, Yeargin-Allsopp, Decouflé \& Murphy, 1995; Yeargin-Allsopp, Decouflé \& Murphy, 1995; Rosenzweing, 1996; Romski \& Sevcik, 1997; Miolo, Chapman \& Sindberg, 2005).

Entonces, el lenguaje no verbal es más que un accesorio del lenguaje, pasa a ser una forma de comunicación, en ocasiones la única opción, que transmite significado. Por lo tanto, lo extralingüístico se transforma en una herramienta útil en la enseñanza para la inclusión de todos los estudiantes, considerando que las diversas demandas que exigen los actuales sistemas educativos, ameritan un trabajo creativo, para que desde el currículum escolar se generen respuestas a las necesidades que presentan los educandos, brindando la posibilidad de aprovechar al máximo su potencial en la perspectiva de lograr personas más íntegras que puedan desarrollar sus proyectos de vida plenamente, privilegiando la igualdad de oportunidades y estableciendo el aula regular como un espacio idóneo para cumplir estos fines (Muñoz., col. 2009).

Además es importante resaltar que la visión multidimensional para atender a los NNA con discapacidad intelectual se ha convertido en un reto para el sistema educativo colombiano actual. De acuerdo a la información presentada en el estudio de Situación de la Educación en Colombia, Preescolar, Básica, Media y Superior, (Sarmiento, 2010), indica que en el Censo nacional de población DANE 2005, las personas con discapacidad son el 6,19 por cada 100 habitantes con un total de 2.549.153 personas con discapacidad; dentro de los departamentos que tienen una proporción por encima de la media nacional corresponden a los departamentos de Cauca, Nariño, Boyacá y Huila. En este censo también se muestra que la proporción por rangos de edad en el censo 2005 de personas con discapacidad muestra que existen sesgos por grupo de edad y sexo. El 16,2\% son niños y niñas hasta los 17 años.
Asimismo, en materia legal ya se han dado los primeros pasos para la integración social de la población con diferentes tipos de discapacidad, acción que se puede evidenciar en los artículos $13^{\circ}, 47^{\circ}, 54^{\circ}$ y $68^{\circ}$ de la Constitución Nacional, la Ley 361 de 1997 y el Decreto No. 366 de 2009. Por medio de este último, se «reglamenta la organización del servicio de apoyo pedagógico para la atención de los estudiantes con discapacidad y con capacidades 0 con talentos excepcionales en el marco de la educación inclusiva» (Col. Decreto No. 366 de 2009).

A pesar de contar con la reglamentación jurídica, el Sistema Educativo Colombiano no cuenta con herramientas e instrumentos sistemáticos de evaluación que le permita conocer cómo aprenden y procesan la información los NNA con discapacidad intelectual, lo que pretende generar adaptaciones curriculares y direccionar estrategias pedagógicas pertinentes que faciliten el aprendizaje y la inclusión educativa de estos estudiantes (Muñoz., col. 2009).

En este contexto y ante la necesidad de utilizar estrategias alternativas para evaluar NNA con discapacidad intelectual, se opta por el modelo de evaluación dinámica y/o potencial de aprendizaje aplicado a una población de NNA de la ciudad de Neiva de manera que se identifique el beneficio que tendrían estos en la utilización de pruebas no verbales, pero sobre todo con el apoyo de un instructor. Esto supondría facilitar y enriquecer los procesos de enseñanza-aprendizaje de estos niños y adolescentes en el contexto escolar.

\section{Metodología}

\section{Tipo de estudio}

Teniendo en cuenta que el objetivo del presente estudio es evaluar el potencial de aprendizaje en niños, niñas y adolescentes con discapacidad intelectual, se opto por un enfoque cuantitativo, con un diseño de tipo pre - experimental pretest y postest a grupo único.

\section{Población}

La población involucrada fueron 17 niños, niñas y adolescentes en edades comprendidas entre 8 y 18 años, escolarizados en la Fundación Síndrome de Down del Huila y el Colegio Utrahuilca de la ciudad de Neiva, todos 
con diagnósticos de Retardo Mental Leve a Moderado e incluidos en los grados de primero a séptimo.

\section{Criterios de inclusión exclusión}

Todos los participantes debían tener un diagnostico previo de discapacidad intelectual entre leve y moderada, estar en edades entre los 8 y 18 años, estar vinculados en el sistema educativo desde el modelo incluyente. En cuanto a los criterios de exclusión se considero que los participantes no estuvieran diagnosticados con algún trastorno psiquiátrico y fallas severas de comprensión de órdenes.

\section{Técnicas e instrumentos}

Los instrumentos se utilizaron de acuerdo a las necesidades del estudio: inicialmente se evaluó inteligencia no verbal y para ello se tomo el TONI-2 (Brown, Sherbenou, \& Johnsen, 2000), el cual mide el funcionamiento intelectual a través de la evaluación de la capacidad para resolver problemas abstractos de tipo gráfico, eliminando la influencia del lenguaje y de la habilidad motriz, la justificación para la utilización de este instrumento es conocer el coeficiente de inteligencia no verbal para determinar la participación en el estudio. Este instrumento muestra al sujeto contenido de tipo abstracto-figurativo en el que debe señalar por medio de una indicación motriz la respuesta que encaja en el espacio vacío. Está compuesto por dos formas equivalente A y B, para este caso se aplicó la forma $A$, ya que se sugiere utilizar en sujetos de muy pocos años en quienes se sospecha deterioro mental significativo y los cuales presentaron efecto techo (si tiene 5 respuestas erróneas consecutivas) antes de completar todo el test de 55 elementos. Todos estos criterios aplicados a los participantes de este tipo de estudio.

Una vez seleccionados los participante, de acuerdo al nivel de inteligencia no verbal, se administró el Test de Matrices Progresivas de Color Raven, series A, Ab y B, ya que este test es exigido por la Evaluación del Potencial de Aprendizaje EPA-2, en las fases pretest-postest. Este permite ser un indicador de la modificabilidad de un sujeto y por tanto, de que presumiblemente va a beneficiarse de un entrenamiento cognitivo, además para obtener el potencial de aprendizaje de un sujeto se puede estimar partiendo de la puntuación que obtiene en el pretest, que supone es la expresión de su capacidad intelectual basal. El Raven fue diseñado para ser utilizado en la investigación de los orígenes genéticos y ambientales de la deficiencia mental. Intenta medir dos componentes del factor $\mathrm{G}$ (factor general de la inteligencia): la capacidad eductiva y la reproductiva. La capacidad eductiva supone una actitud para dar sentido a lo confuso, para forjar constructos no verbales que faciliten el manejo de la complejidad. La aptitud reproductiva atañe a la familiaridad con el acervo de información explícita, en gran medida verbal, de una cultura. La capacidad eductiva envuelve una variedad de procesos perceptuales y conceptuales que se apoyan unos contra otros y suponen tanto procesos afectivos como conativos. A través del proceso deductivo el sujeto extrae nuevas comprensiones e informaciones partiendo de lo que se percibe 0 ya es bien conocido (Raven J. C., 1996:3).

Para la fase intermedia (pretest-postest) se suministró un programa de entrenamiento acompañado de estrategias de mediación significativas con el apoyo del lenguaje no verbal, utilizando la Evaluación del Potencial de Aprendizaje EPA-2, con el cual se persiguió predecir, en los participantes, el grado de modificabilidad cognitiva en un entrenamiento intelectual de larga duración dirigido al aprendizaje de estrategias, procesos o utilización de instrumentos necesarios para la resolución de problemas cognitivos, (Fernández-Ballesteros, Calero, Campllonch \& Belchí, 2010). Este test está compuesto por figuras matriciales (tipo raven) en las cuales el sujeto debe escoger la respuesta correcta que encaje en el diseño estímulo, si la respuesta ofrecida es totalmente incorrecta, se facilitará información sobre las razones de su incorrección hasta que llegue a la resolución de esta, entonces se presenta el diseño - respuesta que actúa como una retroalimentación positiva para el participante.

Este entrenamiento se realizó durante una semana aplicándose en el mismo lugar y hora, se llevo a cabo en tres sesiones; en la primera se utilizaron los diseños del 1 al 18 y en la segunda del 19 al 39 como lo indica el manual de aplicación. En la última sesión se hizo un repaso de los ejercicios anteriores los cuales tuvieron mayor dificultad. También en esta fase se tomaron los registros del proceso de entrenamiento (observaciones semi -estructuradas).

En la Fase de evaluación, postest, se volvió a valorar con el Test de Matrices Progresivas Raven, series A, Ab y $B$, para conocer finalmente el potencial de aprendizaje.

\section{Resultados}

El proceso de búsqueda de los participantes inicio con la revisión de las bases de datos que fueron 
proporcionadas por cada institución. Los participantes se dividieron en dos etapas: Niños (de 8 a 11 años) los cuales eran 4 niños y 1 niña (12 a 18 años) siendo 12 adolescentes varones como lo muestra la Tabla 1, la cual muestra la caracterización de la población. La media de la edad de los participantes fue de 13 años.

Los participantes están escolarizados en los grados de transición a séptimo. Se clasificaron igualmente por escolaridad; un niño cursa Transición; en primaria hay 6 sujetos en primero, 1 en segundo, 1 en cuarto y 2 en quinto; en secundaria están 6 en sexto y 1 en séptimo, como lo muestra la tabla anterior.

Con respecto a la evaluación para conocer las características del funcionamiento intelectual de base de los participantes, se pudo observar a través Test de Inteligencia No Verbal TONI-2, que los participantes del estudio están ubicados en un $\mathrm{Cl}$ (Cociente Intelectual) medio, medio bajo y bajo. El CI Medio esta comprendido entre 90 y 110, lo que correspondió a 4 participantes con edades comprendidas entre los 9 y 13 años. En el Cl Medio Bajo (80-89) se ubicaron 5 NNA en edades entre 10 y 18 años; el $\mathrm{Cl}$ Bajo (70-79) incluyo a 4 NNA, cuyas edades oscilaban entre 8 y 16 años, y por último, en el $\mathrm{Cl}$ Muy Bajo (>70) se ubicaron 4 participantes en edades entre 11 y 16 años. Lo anterior corresponde positivamente con la interpretación que da el Toni-2 para las puntuaciones que estuvieron por debajo de la media de los cocientes de desviación (100), lo que puede indicar retraso mental, problemas de desarrollo u otros trastornos cognitivos.

Con respecto a los datos arrojados por la EPA, el cual recordamos, persigue la predicción del grado de modificabilidad de un sujeto en un entrenamiento intelectual de larga duración, según el manual del mismo, el procedimiento conlleva a la aplicación del Test Matrices Progresivas (escala color) de Raven, antes y después del entrenamiento. Luego de su corrección, se tendrán dos puntuaciones directas, una anterior al entrenamiento 0 puntuación pre-test y la otra posterior al entrenamiento 0 puntuación post-test. Así, la diferencia entre estas dos puntuaciones (pos-test-pre-test) será considera Puntuación Directa de Ganancia (PDG) y esta puntuación será utilizada como criterio de ganancia diferencial entre sujetos «ganadores» 0 «no ganadores» en un punto de corte establecido de 5 puntos de ganancia (Fernández-Ballesteros, Calero, Campllonch \& Belchí, 2010).

Lo anterior permite soportar los resultados arrojados en este estudio y plasmados a continuación en la Tabla 2 , en la cual se observa los resultados en la pruebas Raven pretest y postest y el total de Puntuación Directa de Ganancia PDG, el mismo que sería el Potencial de Aprendizaje obtenido por los participantes; a nivel general, se observó que la media de los resultados obtenidos

Tabla 1. Caracterización de la población

\begin{tabular}{|c|c|c|c|c|c|c|c|c|}
\hline \multirow[t]{2}{*}{ Grupo } & \multirow[t]{2}{*}{$\mathrm{N}$} & \multicolumn{2}{|c|}{ Lateralidad } & \multicolumn{2}{|c|}{ Sexo } & \multirow{2}{*}{$\begin{array}{l}\text { Edad media } \\
\text { (DE) }\end{array}$} & \multirow{2}{*}{$\begin{array}{l}\text { Escolaridad media } \\
\text { (DE) }\end{array}$} & \multirow{2}{*}{$\begin{array}{l}\text { TONI-2 Cl Total media } \\
\text { (DE) }\end{array}$} \\
\hline & & D & I & M & $\mathbf{F}$ & & & \\
\hline Estudio & 17 & 12 & 5 & 16 & 1 & $\begin{array}{c}13 \\
(2,7)\end{array}$ & $\begin{array}{c}3,2 \\
(2,4)\end{array}$ & $\begin{array}{c}79,5 \\
(10,3)\end{array}$ \\
\hline
\end{tabular}

Tabla 2. Raven pre - postest y potencial directo de ganancia (PDG)

\begin{tabular}{|c|c|c|c|c|c|c|}
\hline \multicolumn{7}{|c|}{ Descriptivos } \\
\hline & & Mediana & Mínimo & Máximo & Rango int & $\begin{array}{l}\text { (Puntuación Directa de Ganancia) } \\
\text { Media }\end{array}$ \\
\hline Raven & $\begin{array}{l}\text { Pretest } \\
n=17 \\
\text { Postest } n=17\end{array}$ & $\begin{array}{l}16 \\
17\end{array}$ & $\begin{array}{c}4 \\
13\end{array}$ & $\begin{array}{l}31 \\
33\end{array}$ & $\begin{array}{l}27 \\
20\end{array}$ & 3,3 \\
\hline
\end{tabular}


en el PDG, 3.3, refiere que la mayoría de los participantes no tuvieron una ganancia significativa, razón por la cual pudieron haber otros factores existentes que influenciaron los mismos. Descriptivamente, aparecen seis participantes con edades de 8,10, dos de 13 y dos con 14 años, que cuentan con una escolaridad de primero de primaria (3 sujetos) y sexto de bachillerato (3 sujetos), quienes en sus puntuaciones reflejan una ganancia significativa en el potencial de aprendizaje (PDG de 6, dos de 7, 8, 13 y 16), estos seis sujetos, aumentaron las respuestas correctas en el Raven postest luego de aplicar el entrenamiento, en el cual se observó que a pesar de que la mayoría de las respuestas de los niños de baja escolaridad fueron impulsivas (contesta al azar) teniendo que hacer una corrección tras la intervención del investigador, los otros las resolvieron de forma sistemática (el sujeto examina el problema detenidamente teniendo en cuenta todas las variables que intervienen en el diseño) (Fernández Ballesteros R, Calero MD, Campllonch JM, Belchí, 2010). Las características del funcionamiento intelectual de base de estos participantes, $\mathrm{Cl}$ de Bajo a Medio (Cl: 76;79;82; 83;89 y 91), y el nivel de compromiso por su condición de discapacidad, muestran que incorporaron estrategias de aprendizaje útiles para su desempeño en los problemas cognitivos de tipo abstracto-figurativo, lo que permite ser un buen predictor de las mejoras que ocurran tras un entrenamiento de amplio alcance en habilidades intelectuales.

Por otra parte, hubo puntuaciones ubicadas en un PDG entre 0 y 2 ( 6 sujetos con edades entre los $9,12,15$ y 16 años y escolaridad entre $2^{\circ}, 3^{\circ}, 4^{\circ}, 6^{\circ}$ y $7^{\circ}$ ), es decir, obtuvieron un bajo potencial de aprendizaje, lo que permite inferir que a pesar del entrenamiento implementado con la EPA, sus resultados no fueron significativos 0 ventajosos para emitir una solución adecuada a dichos problemas, ya que se observaba que respondían de forma impulsiva, manteniendo un patrón de respuesta fija, es decir, a medida que se le mostraban las figuras de entrenamiento diseño - estímulo, siempre respondían de la misma manera que la respuesta anterior, evidenciando compromiso en la flexibilidad mental y la inhibición. Sin embargo, ello no debería determinar su exclusión de una intervención cognitiva posterior.

Por último, 5 participantes tuvieron respuestas negativas, es decir, las respuestas dadas en el pretest fueron mayores que las obtenidas en el postest, lo que refleja, como lo dicho anteriormente, un bajo potencial de aprendizaje que igualmente no están exentos de recibir una intervención cognitiva en un futuro que facilite un aprendizaje de una serie de procesos y operaciones ejecutivas de control requeridas para el funcionamiento intelectual. Estos sujetos, tienen edades comprendidas entre los 11 y 16 años y una escolaridad entre $1^{\circ}$ y $5^{\circ}$ de primaria, reflejan en sus respuestas un compromiso impulsivo y no sistemático a la hora de dar solución a los problemas. A pesar de que la mayoría tienen una edad cronológica alta, su escolaridad es muy baja, sumado a que 4 de los 5 participantes tienen un $\mathrm{Cl}$ Muy Bajo (>70) y uno con $\mathrm{Cl}$ Medio ( 90 y 110), lo que correlaciona con un diagnóstico de discapacidad intelectual moderada, reflejando que estos datos pueden de alguna manera justificar las puntuaciones bajas en las pruebas, pero teniendo en cuenta que hay otros factores (condiciones biológicas 0 motivacionales) que pudieron estar relacionados con la ejecución en sus respuestas.

\section{Discusión}

El presente estudio pretendía evaluar la evaluación dinámica y del potencial de aprendizaje de un grupo de 17 niños, niñas y adolescentes con discapacidad intelectual, el cual intentaba modificar las funciones cognitivas considerando los estados emocionales de los NNA, a la hora de dar respuesta a una serie de tareas de características no verbales, y buscando determinar, de acuerdo al soporte teórico, si existe una ganancia en el potencial de aprendizaje en estos participantes sometidos a un entrenamiento intelectual. Estos resultados observados demuestran que en algunos sujetos hubo ganancia en la prueba EPA-2, recordando que una ganancia es significativa a partir de 5 puntos, y las obtenidas por los sujetos de la presente investigación estuvieron comprendidas entre 6 y 16 puntos. Investigaciones realizadas en otros países como España (Robles \& Calero, 2008) y Sur África (Skuy, Gewer, Osrin, Khunou, Fridjhon \& Rushton, 2002) soportan estos hallazgos, en los cuales demuestran la ganancia que presentan los sujetos en el potencial de aprendizaje cuando los mismos son evaluados en un contexto de evaluación dinámica.

Los resultados anteriores corroboran la existencia de modificabilidad cognitiva en niños y adolescentes a pesar de su discapacidad intelectual (Budoff, 1974; Calero, 2004; Brown \& Campione, 1992; Carlson \& Wiedl, 2000; Fernández-Ballesteros \& Calero, 2000; Hessels \& Hamers, 1993; Lauchlan \& Elliot, 2001; Lidz \& Thomas, 1987; Tzuriel \& Klein, 1987); lo cual resulta importante mencionar te- 
niendo en cuenta que el tipo de intervención que reciben los mismos en sus respectivas instituciones educativas evidencia la permanencia de la vieja concepción de las personas en situación de discapacidad desde lo psicopatológico (Verdugo, 2003a) o desde el referente de la normalidad, lo cual no facilita la incorporación de aprendizajes y alcance en habilidades intelectuales ni a la intervención dada a la población evaluada.

Confirmando lo anterior, de que pesar de que una de las instituciones escolares tiene inclusión educativa, se observa la continua práctica de esta evaluación tradicional en los participantes de la investigación, coincidiendo que resulta evidente el desconocimiento de la evaluación dinámica como alternativa pertinente para la evaluación de personas con discapacidad cognitiva (Guthke \& Stein, 1996); planteando con ello que el instrumento de evaluación debe acomodarse siempre a las características individuales de los sujetos.

Por otro lado, no podemos referir que los otros sujetos que obtuvieron un bajo potencial de aprendizaje no aprovecharon el entrenamiento cognitivo, al contrario, de alguna manera se logró una estimulación cognitiva, pero que debido a la complejidad del compromiso cognitivo, atencional y/u otros factores motivaciones, pudieron afectar la resolución de los problemas de tipo abstractofigurativo. Se observó que los niños en los cuales presentaban una discapacidad cognitiva compleja, mostraron dificultades en atención y compresión de los diseños, ya que la mayoría contestaban de forma impulsiva de acuerdo a la anterior solución manteniendo un patrón de respuesta fija, evidenciando compromiso en la flexibilidad mental y la capacidad de inhibición. Su nivel de atención se mantenía en un período muy corto, ya que en las últimas respuestas no lograban discriminar entre las distintas opciones sino que mantenían este patrón sistemático, pudiendo esto estar relacionados con los bajos resultados obtenidos.

Igualmente este estudio facilitó el uso de técnicas y estrategias (TONI-2, RAVEN), que apuntan hacia la comunicación no verbal, confirmando la importancia que tiene este a la hora de evaluar y abordar esta población, en donde el aprendizaje de niños con estas características, parte desde lo más procedimental (habilidades perceptivas, motoras y cognitivas adquiridas, no conscientes, relacionadas con la acción) hacia lo declarativo (más explícito, referido a las habilidades y hechos que se pueden expresar de manera consciente), y combinando el uso de lenguaje verbal con lenguaje no verbal (Seys, Kersten \& Duker, 1990; Romski \& Sevcik, 1997; Buffington, Krantz, McClannahan \& Poulson, 1998; Smith \& Bryson, 1998; Wetherby, Prizant \& Hutchinson, 1998; Jones \& Carr, 2004; Stephenson \& Dowrick, 2005), además que la comunicación no verbal facilita los procesos de atención y memoria que influyen en la interpretación y comprensión de la información por parte de estos sujetos (Drews, Yeargin-Allsopp, Decouflé \& Murphy, 1995; YearginAllsopp, Decouflé \& Murphy, 1995; Rosenzweing, 1996; Romski \& Sevcik, 1997; Miolo, Chapman \& Sindberg, 2005). Sin embargo, y desafortunadamente las escalas con importante contenido verbal continúan siendo utilizadas en diferentes investigaciones con propósitos clasificatorios, a pesar de dichas dificultades (Calero \& Robles Bello, 2010).

Por último, hay que recalcar que con estos resultados se provee un estudio que confirma la importancia de que el entrenamiento adecuado a los menores con discapacidad intelectual puede ser predictor en la mejora que puede ocasionar tras un tratamiento de amplio alcance en las habilidades intelectuales, además de considerar una propuesta de cambio del paradigma de la evaluación tradicional a la evaluación dinámica que hasta el momento es eficaz en esta población.

\section{Conclusiones}

Se observan diferencias en los resultados del potencial de aprendizaje en el grupo de niños participantes. Para algunos participantes se considera hubo efectividad del entrenamiento observado esto en los resultados favorables, en especial en el aprendizaje de una serie de procesos y operaciones ejecutivas requeridos en la resolución de este tipo de problemas. Por otro lado, hay niños, los cuales por su compromiso cognitivo moderado que involucra dificultad en la flexibilidad mental y capacidad de inhibición, además de dificultades atencionales y factores motivaciones, entre otros, no se vieron beneficiados de este entrenamiento, sin embargo, esto no descarta que la incorporación de estrategias especificas y con un entrenamiento más amplio se logre estimular otras áreas y habilidades intelectuales.

Una ventaja de este paradigma, como lo plantea Calero (2004), es que se utiliza en sujetos con posibilidad de mejora; su objetivo no es medir tan solo la ejecución de los sujetos, sino su posibilidad de aprendizaje, y esta ca- 
racterística se pudo evidenciar en la presente investigación porque algunos sujetos obtuvieron ganancia significativa en el potencial de aprendizaje (PDG entre 6 y 16) y el punto de corte en la ganancia entre pre-test y postes es de 5 PDG (Ballesteros, Calero, Campllonch \& Belchí, 2010).

Todavía queda mucho por indagar y estudiar en este nuevo paradigma de evaluación dinámica. Se tiene la expectativa, con el apoyo de los resultados obtenidos en esta investigación, de que la evaluación del potencial de aprendizaje sea una alternativa empleada y esté al alcance de los profesionales que trabajan con esta población en situación de discapacidad intelectual y que además la intervención incluya la atención individualizada y observación de otros factores que puedan influir en la ejecución de los resultados, ya que hay que tener en cuenta que, comprender cómo aprenden y procesan la información los niños, niñas y adolescentes con discapacidad intelectual es crucial para poder generar adaptaciones curriculares pertinentes en el proceso de enseñanzaaprendizaje, esperando que se modifiquen las prácticas pedagógicas docentes con el fin de facilitar dichos aprendizajes, partiendo desde las capacidades y atributos individuales de estos niños.

Finalmente, el presente estudio tiene la limitación de contar con una muestra reducida (diecisiete sujetos), esto debido a las condiciones mismas en las que se encuentra la población con discapacidad intelectual que se haya en la región, en la misma no se cuenta con programas de atención y ubicación a las personas con discapacidad, y los sitios que existen en donde se integran a los niños en un espacio físico común, hay ausencia de herramientas clínicas y educativas para el diagnostico y abordaje adecuado para ellos.

Por lo tanto se recomienda que esta metodología investigativa se replique en una muestra representativa de la población para corroborar los hallazgos evidenciados en este estudio.

\section{Referencias bibliográficas}

Brown, Ann L. y Joseph C. Campione. «Inteligencia académica y capacidad de aprendizaje». En ¿Qué es la inteligencia? Enfoque actual de su naturaleza y definición, de Douglas K. Detterman y Robert J. Sternberg, 57-62. Madrid: Ediciones Pirámide, 1992.
Brown, Linda, Rita J. Sherbenou y Susan K. Johnsen. 2000. Test de inteligencia no verbal TONI-2. Madrid: TEA ediciones.

Budoff, M.; Gimon, A. y Corman, L. «Learning Potential Measurement with Spanishs peaking Young as an Alternative to I.Q Tests: A First Report». Studies in Learning Potential, 70, Cambridge MA, RIEP, 1975.

Buiza-Navarrete, J. J., J. A. Adrián-Torres, M. GonzálezSánchez y M. J. Rodríguez-Parra. «Evaluación de marcadores psicolingüísticos en el diagnóstico de niños con trastorno específico del lenguaje». Revista de Logopedia, Foniatría y Audiología. 24(4)(2004):142-156.

Calero MD, Márquez J. Psychometric Properties of a Learning Potential Test for Reading: The Picture Word Game. Europ J Psychol Assess, 14(1998):124-133.

Fernández Ballesteros R, Calero MD, Campllonch JM, Belchí. Instrumento para la Evaluación del Potencial de Aprendizaje: EPA. Madrid: MEPSA, 1987/1990.

Fernández Ballesteros R, Calero MD. Nuevos enfoques en la medida de la inteligencia: la Evaluación del Potencial de Aprendizaje. En Inteligencia y Cognición: Homenaje al Profesor Yela. Madrid: Ed. Complutense, (1992):546-590.

Fernández Ballesteros R, Juan-Espinosa M de, Colom $R$, Calero M D. Contextual and personal sources of individual differences in intelligence: some empirical results. Adv Cogn Educ Practice, 4:(1997)221-274.

Feuerstein R, Rand Y. Mediated learning experiences: an outline of the proximal etiology for differential development of cognivive functions. International Understanding, L. Gold Fein (ed), 1974:9/10:7-37.

Gutiérrez-Clellen, V. F. «La evaluación dinámica del lenguaje». Revista Chilena de Fonoaudiología, 4(1)(2003): 51-59.

Guthke, J., y H. Stein. «Are learning tests the better version of intelligence tests?» European Journal of Psychological Assessment, 12(1)(1996):1-13.

Gooding Londoño, M. P. Proyecto: evaluación del potencial de aprendizaje del sistema ejecutivo, desde el 
modelo de evaluación dinámica en los niños y niñas con discapacidad intelectual. Universidad Surcolombiana, 2010.

Haywood, y D. Tzuriel. Interactive assessment. New York: Springer-Verlag, 1992.

Lauchlan, F., y J. Elliot. «The psychological assessment of learning potential». British Journal of Educational Psychology, 71(2001):647-665.

Lidz, C.S., y C. Thomas. «The preschool learning assessment aevice: extension of a atatic approach». En Dynamic assessment: An interactional appoach to evaluating learning potential, de C. Lidz, 123-164. New York: The Guilford Press, 1987.

Luckasson, R., Borthwick-Duffy, S., Buntix, W.H.E., Coulter, D.L., Craig, E.M., Reeve, A., y cols. Mental Retardation. Definition, classification and systems of supports (10th ed.). Washington, DC: American Association on Mental Retardation. [Traducción al castellano de M.A. Verdugo y C. Jenaro (en prensa). Madrid: Alianza Editorial], 2002.

Márquez J. Validación del P.W.G.: un test de Potencial de Aprendizaje para la lectura. Tesis Doctoral. Facultad de Psicología. Universidad de Granada, 1995.

Muñoz, María T., González Carlos., Lucero Boris. Influencia del lenguaje no verbal (gestos) en la memoria y el aprendizaje de estudiantes con trastornos del desarrollo y discapacidad intelectual: Una revisión. Universidad Católica del Maule Chile. Revista Signos, 42(69)(2009):29-49.

Raven, J. C. Test de Matrices Progresivas. Manual, Buenos Aires, Paidós, 1996.
Robles, M. A., y M. D. Calero. «Evaluación de funciones cognitivas en la población con síndrome de Down». Revista de Síndrome de Down: Revista española de investigación e información sobre el Síndrome de Down, núm. 97(2008):56-66.

Skuy, Mervyn, Anthony Gewer, Yae I Osrin, David Khunou, Peter Fridjhon, y J. Philippe Rushton. «Effects of mediated learning experience of raven's matrices scores of african and non-african university students in South Africa». Intelligence, 30(2)(2002):221-232.

Sternberg, R. J. «Cognitivelehavioral Approaches to the Training of Intelligence in the Retarded». J. of Special Education, 15(1981):165-183.

Trilla, J. El legado pedagógico del siglo XX para la escuela del siglo XXI. Lev S. Vogotsky: la psicología cultural y la construcción de la persona desde la educación. España: Editor Grao. ISBN: 8478272569,9788478272563, p. 219, 2001.

Tzuriel, D. «Dynamic assessment is not dynamic testing». Issues in Education, 7(2)(2001):237-250.

Vásquez Barrios, Armando J. Proyecto de salud mental. Organización panamericana de la salud, Santiago de Chile, Chile En: Rodríguez, J.; Kohn, R. y Aguilar, S. Epidemiología de los trastornos mentales en América Latina y el Caribe. Washington, D.C.

Verdugo Alonso, M.A.; Aguado Díaz, A. L. Y Aguado, A. Personas con discapacidad: perspectivas psicopedagógicas y rehabilitadoras. Ed. ilustrada. Editor siglo XXI de España Editores. ISBN 8432308676, 9788432308673, 556-559 p., 1995.

Vygotsky LS. El desarrollo de los procesos psicológicos superiores. Barcelona: Grijalbo, 1978. 